\title{
Combining old and new strategies for colorectal cancer screening
}

\author{
Elisa Danese, Martina Montagnana, Giuseppe Lippi \\ Section of Clinical Biochemistry, University of Verona, Verona, Italy \\ Correspondence to: Dr. Elisa Danese. Section of Clinical Biochemistry, University Hospital of Verona, Piazzale LA Scuro, 37134 Verona, Italy. \\ Email: elisa.danese@univr.it. \\ Provenance: This is an invited article commissioned by the Editorial Office of Annals of Translational Medicine. \\ Comment on: Fang Y, Xiao B, Peng J, et al. An early report of a screening program for colorectal cancer in Guangzhou, China. \\ Ann Transl Med 2019;7:604.
}

Submitted Nov 16, 2018. Accepted for publication Nov 25, 2019.

doi: 10.21037/atm.2019.11.135

View this article at: http://dx.doi.org/10.21037/atm.2019.11.135

Colorectal cancer (CRC) is the third most common form of cancer, accounting for approximately $10 \%$ of all cancerrelated deaths worldwide and accounting for as many as 19.1 million cause-specific disability-adjusted life years (DALYs) each year (1). The highest incidence rate is found in developed countries and seems prevalently associated with western lifestyle and dietary habits. However, the epidemiologic burden of CRC is raising also in other parts of the world, such as in Asia, where CRC incidence has increased by 2 - to 4 -fold during the past decade (1).

Due to the concerning incidence rate and the high grade of associated disability and mortality, CRC represents now a major public health issue all around the world. Due to the slow progression from detectable precancerous adenoma polyps to neoplastic lesions and to the favorable prognosis of patients in whom the disease can be diagnosed at an early stage, development and implementation of effective screening programs shall be regarded as a major health goal $(2,3)$. According to the principle guidelines for CRC screening, first-line test should be based on detecting the presence of blood in the stool, since this approach was proven as being cost-effective, is naturally noninvasive and allows good accessibility and patient compliance. Among the available options, guaiac-based fecal occult blood tests (gFOBT) and immunochemical fecal occult blood tests (FIT) now represent the most widely used stool assays for initial screening. A systematic review of randomized control trials and observational studies has recently showed that FIT may be more effective than gFOBT for reducing CRC mortality. In this meta-analysis the efficacy of gFOBT versus no screening was assessed in 19 studies including more than 2 million participants, whilst the efficacy of FIT versus no screening was explored in 4 studies including over 5 million participants. Overall, gFOBT was associated with a $14 \%$ decreased mortality for CRC, whereas CRC deaths in average-risk populations could be reduced by $59 \%$ using FIT (4). The estimated effect was $79 \%$ reduction in CRC mortality when screening with FIT was compared with annual or biennial gFOBT. This was mainly attributed to the better clinical and analytical performance of immunochemical technique compared with gFOBT. Pooled data extracted from 19 different studies confirmed by colonoscopy or 2-year follow-up revealed that global sensitivity and specificity of FIT for CRC are as high as 0.79 [95\% confidence interval (95\% CI), 0.69-0.86] and 0.94 (95\% CI, 0.92-0.95), respectively (5). Based on a single meta-analysis, FIT was found to perform better that $\mathrm{gFOBT}$ for detecting both CRC [relative risk (RR), 1.96; 95\% CI, 1.2-3.2] and advanced neoplasia (RR, 2.28; $95 \%$ CI, 1.68-3.10), with no impact on specificity (6). The superiority of FIT over gFOBT is mainly due to the fact that gFOBT is based on detection of the peroxidaselike activity of the heme group on guaiac acid, which is vulnerable to many dietary interferences. Unlike gFOBT, FIT encompasses the use of antibodies specifically reacting with human hemoglobin, so that the assay is predictably less vulnerable to dietary (e.g., animal-derived hemoglobin or myoglobin) and drugs interference (e.g., nonsteroidal antiinflammatory drugs). Therefore, gFOBT does not allow a straightforward distinction between upper and lower gastrointestinal tract bleedings, whilst FIT is much more selective for occult bleeding of colorectal origin, whereby 
hemoglobin from upper gastrointestinal tract is rapidly digested in stomach and small intestine. Due to its higher sensitivity, FIT only needs one sample rather than three specimens collected in three separate days, as necessary for gFOBT. Less vulnerability to several preanalytical factors and major patients' compliance to screening are other wellknown advantages of FIT compared to gFOBT (7-9).

As concerns the higher adherence to screening, a randomized trial based on patients invited to participate in a CRC screening program based on either FIT or gFOBT showed that the participation rate was significantly higher for FIT compared to gFOBT (i.e., $61 \%$ versus $49 \%$ ) (10). Another recent study, in which the adherence rate of a pilot biennial FIT-screening program was assessed, demonstrated that participation was as high as $63 \%$ over four separate rounds (11). Similar participation rates, between $56 \%$ and $63 \%$, have been reported in a pilot study based on over four rounds of biennial FIT screening in Italy (12). Although these studies indicate that replacing gFOBT with FIT may lead to considerable increase of both adherence to CRC screening program and CRC detection rate, the proportion of incompliant patients remains clinically high (i.e., almost one fourth).

In an article published in this issue of Annals of Translational Medicine, Fang and coauthors describe the possible benefits of a new screening program based on two FIT tests combined with a questionnaire designed for specifically identifying subjects at higher risk of CRC and encompassing a vast array of clinical information such as age, sex, familial or personal history of cancer, gastrointestinal symptoms, dietary habits and cigarette smoking (13). Among 1,219 candidates for colonoscopy (out of 6,971 invitees), only 647 (53.1\%) were finally compliant. A percentage as high as $77.1 \%$ of these subjects was categorized as positive by the questionnaire, whilst 36.8\% of all subjects tested positive with FITs, thus suggesting that the self-evaluating questionnaire may generate better benefits than laboratory-assessed FIT in persuading subjects on the need of undergoing colonoscopy. As regards the predictive performance of this strategy, the combination of the questionnaire with FITs improved the diagnostic sensitivity by over $40 \%$, whilst concomitantly decreasing the diagnostic specificity by $58 \%$. More specifically, the diagnostic sensitivity of the questionnaire was 0.58 , that of FITs was 0.47 , whilst that of their combination increased to 0.92 . On the other hand, the diagnostic sensitivity of the questionnaire was 0.33 , that of FIT was 0.67 , whilst their combination yielded a diagnostic specificity as low as 0.09. This screening program may hence be effective for identifying patients with colonic neoplasms (mostly at an early stage), whilst it would be contextually associated with a considerably large proportion of healthy subjects who may be addressed to unnecessary colonoscopy.

Since screening with flexible sigmoidoscopy and colonoscopy seems the most straightforward means for detecting early and advanced colon lesions, the development of non-invasive screening approach capable to identify high risk patients who may need to be referred to invasive inspection remains challenging (14). Ideally, the screening program should be broadly accepted by the general population, be characterized by high sensitivity and specificity, but shall also be safe, widely available and costeffective. FIT test has achieved a good level of acceptability in the general population, but still lacks optimal diagnostic performance. To reduce costs and improve sensitivity, the combination of FIT with a high-risk screening questionnaire may be seen as a feasible and inexpensive option. The current evidence provided by Fang et al. (14) seemingly suggests that this approach may help decreasing the false negative rate characterizing FIT, thus confirming earlier literature data (15), Nevertheless, the dramatically low diagnostic specificity raises doubts as to whether such strategy may be really clinically and economically sustainable, especially considering that the sum of its sensibility and specificity is lower than that of FIT alone (1.007 vs. 1.148). Long-term data are hence needed for assessing the real clinical, social and economic benefits of this innovative strategy, perhaps administered with cognitive interviewing methodology for improving the accuracy and reliability of responses in certain categories of subjects.

\section{Acknowledgments}

None.

\section{Footnote}

Conflicts of Interest: The authors have no conflicts of interest to declare.

Ethical Statement: The authors are accountable for all aspects of the work in ensuring that questions related to the accuracy or integrity of any part of the work are appropriately investigated and resolved. 


\section{References}

1. Mattiuzzi C, Sanchis-Gomar F, Lippi G. Concise update on colorectal cancer epidemiology. Ann Transl Med 2019;7:609.

2. Danese E, Montagnana M. Epigenetics of colorectal cancer: emerging circulating diagnostic and prognostic biomarkers. Ann Transl Med 2017;5:279.

3. Danese E, Montagnana M, Lippi G. Circulating molecular biomarkers for screening or early diagnosis of colorectal cancer: which is ready for prime time? Ann Transl Med 2019;7:610.

4. Zhang J, Cheng Z, Ma Y, et al. Effectiveness of Screening Modalities in Colorectal Cancer: A Network MetaAnalysis. Clin Colorectal Cancer 2017;16:252-63.

5. Lee JK, Liles EG, Bent S, et al. Accuracy of fecal immunochemical tests for colorectal cancer: systematic review and meta-analysis. Ann Intern Med 2014;160:171.

6. Hassan C, Giorgi Rossi P, Camilloni L, et al. Metaanalysis: adherence to colorectal cancer screening and the detection rate for advanced neoplasia, according to the type of screening test. Aliment Pharmacol Ther 2012;36:929.

7. Rapi S, Cellai F, Rubeca T. New frontiers in fecal immunochemical and other fecal tests in colorectal cancer screening. J Lab Precis Med 2017;2:88.

8. Spiteri N, Skaife P. Get FIT for the new year: a review of the role of faecal immunochemical test for haemoglobin in patients with symptoms of colorectal disease. J Lab Precis Med 2018;3:52.

9. Gies A, Niedermaier T, Weigl K, et al. Effect of longterm frozen storage and thawing of stool samples on faecal haemoglobin concentration and diagnostic performance of faecal immunochemical tests. Clin Chem Lab Med 2020;58:390-8.

10. Hol L, van Leerdam ME, van Ballegooijen M, et al. Screening for colorectal cancer: randomised trial comparing guaiac-based and immunochemical faecal occult blood testing and flexible sigmoidoscopy. Gut 2010;59:62.

11. van der Vlugt M, Grobbee EJ, Bossuyt PM, et al. Adherence to colorectal cancer screening: four rounds of faecal immunochemical test-based screening. Br J Cancer 2017;116:44-9.

12. Crotta S, Segnan N, Paganin S, et al. High rate of advanced adenoma detection in 4 rounds of colorectal cancer screening with the fecal immunochemical test. Clin Gastroenterol Hepatol 2012;10:633-8.

13. Fang Y, Xiao B, Peng J, et al. An early report of a screening program for colorectal cancer in Guangzhou, China. Ann Transl Med 2019;7:604.

14. Symonds EL, Fraser RJ, Young GP et al. FIT for purpose: enhanced applications for faecal immunochemical tests. J Lab Precis Med 2018;3:28.

15. Baier M, Calonge N, Cutter G, et al. Validity of selfreported colorectal cancer screening behavior. Cancer Epidemiol Biomarkers Prev 2000;9:229-32.
Cite this article as: Danese E, Montagnana M, Lippi G. Combining old and new strategies for colorectal cancer screening. Ann Transl Med 2020;8(4):67. doi: 10.21037/ atm.2019.11.135 